\title{
Postmarketing Trials and Pediatric Device Approvals
}

AUTHORS: Thomas J. Hwang, AB, a,b Aaron S. Kesselheim, MD, JD, MPH, ${ }^{b}$ and Florence T. Bourgeois, MD, MPH ${ }^{c, d}$

aFaculty of Arts and Sciences, Harvard University, Cambridge, Massachusetts; 'bProgram on Regulation, Therapeutics, and Law, Division of Pharmacoepidemiology and Pharmacoeconomics, Department of Medicine, Brigham and Women's Hospital and Harvard Medical School, Boston, Massachusetts; 'Division of Emergency Medicine, Boston Children's Hospital, Boston, Massachusetts; and ${ }^{d}$ Department of Pediatrics, Harvard Medical School, Boston, Massachusetts

\section{KEY WORDS}

medical device, clinical trial, children, pediatric, postmarketing, device safety

\section{ABBREVIATIONS}

FDA—Food and Drug Administration

HDE-humanitarian device exemption

$\mathrm{IQR}$-interquartile range

PMA—premarket approval

PMDSIA—Pediatric Medical Device Safety and Improvement Act of 2007

Mr Hwang conceptualized and designed the study, carried out data collection and analysis, and drafted the initial manuscript; Dr Kesselheim conceptualized and designed the study and critically reviewed the manuscript; Dr Bourgeois conceptualized and designed the study, supervised data collection and analysis, and critically reviewed the manuscript; and all authors approved the final manuscript as submitted.

www.pediatrics.org/cgi/doi/10.1542/peds.2013-3348

doi:10.1542/peds.2013-3348

Accepted for publication Jan 29, 2014

Address correspondence to Florence T. Bourgeois, MD, MPH, Division of Emergency Medicine, Children's Hospital Boston, 300 Longwood Ave, Boston, MA 02115. E-mail: florence.

bourgeois@childrens.harvard.edu

PEDIATRICS (ISSN Numbers: Print, 0031-4005; Online, 1098-4275).

Copyright (c) 2014 by the American Academy of Pediatrics

FINANCIAL DISCLOSURE: The authors have indicated they have no financial relationships relevant to this article to disclose.

FUNDING: Dr Bourgeois was supported by a grant from the National Institute of Child Health and Human Development (1R21HD072382), National Institutes of Health. Mr Hwang received research grants for this study from the Interfaculty Initiative in Health Policy (Cordeiro Research Fellowship) and the Center for American Political Studies, both at Harvard University. Dr Kesselheim was supported by a career development award from the Agency for Healthcare Research and Quality (K08HS18465-01), a Greenwall Faculty Scholarship in Bioethics, and a Robert Wood Johnson Foundation Investigator Award in Health Policy Research.

POTENTIAL CONFLICT OF INTEREST: The authors have indicated they have no potential conflicts of interest to disclose.
WHAT'S KNOWN ON THIS SUBJECT: Medical devices approved for adults can be used to treat children despite the lack of rigorous evidence. In 2007, Congress passed the Pediatric Medical Device Safety and Improvement Act to stimulate pediatric device development.

WHAT THIS STUDY ADDS: Most pediatric devices approved since the legislative change have had limited premarket study in children, with pediatric patients representing $<10 \%$ of trial participants. Postmarketing studies required by the US Food and Drug Administration also yielded limited additional pediatric data.
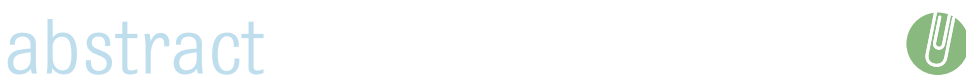

BACKGROUND: Medical devices can be useful in a variety of diseases, but few devices have been specifically approved for use in children. The 2007 Pediatric Medical Device Safety and Improvement Act was passed to stimulate pediatric device development. The current state of trial evidence underpinning the approval of pediatric devices remains poorly described.

METHODS: We identified all high-risk (ie, class III) devices approved through the premarket approval or humanitarian device exemption pathways for therapeutic use in children between 2008 and 2011. We collected key information on clinical trial design (randomization, blinding, controls, and types of end points) as well as age distribution of trial participants. We also identified US Food and Drug Administration (FDA)-mandated postmarketing trials.

RESULTS: Twenty-two devices were approved for use in children via the premarket approval pathway and 3 via the humanitarian device exemption pathway. Twenty-two (88\%) qualified as pediatric despite minimum approval ages of $\geq 18$ years (the FDA Center for Devices and Radiologic Health considers patients 18-21 years old as pediatric). Most devices were approved on the basis of nonrandomized $(59 \%)$, open-label (68\%) studies with surrogate effectiveness end points (86\%). Overall, 21 (84\%) devices were not studied in any patients $<18$ years of age. Postmarketing studies were mandated by the FDA for 19 (76\%) devices, although only 3 (18\%) required enrollment of pediatric patients.

CONCLUSIONS: Most high-risk pediatric devices are approved on the basis of trials in patients $\geq 18$ years old, with few pediatric patients exposed to the devices before market availability. Few postmarketing studies require additional study in pediatric patients. Pediatrics 2014;133:e1197-e1202 
Although medical devices can be lifesaving for a variety of diseases, there are significant challenges associated with their use in children. Few devices have been developed specifically for children. The US Food and Drug Administration (FDA) estimates that pediatric device development lags 5 to 10 years behind that of adult devices. ${ }^{1}$ As a result, clinicians often adapt adult devices, such as stents, defibrillators, and orthopedic implants, for pediatric use. ${ }^{2}$ In some cases, adult devices are reengineered or resized to fit pediatric patients. There are considerable risks associated with this approach given the limited experience and formal assessments of these modified devices, and a number of adverse events linked to adult device use in children have been reported. ${ }^{3-6}$ Another common practice is to use adult devices "off label," or outside that device's FDAapproved indication. In pediatric interventional cardiology, for example, reports have estimated that $>60 \%$ of patients who undergo a therapeutic cardiac catheterization are exposed to off-label use of adult devices.?

The risks of using adult-approved devices in children are particularly salient in the case of high-risk (ie, class III) devices, which are defined asthose that support or sustain life, or that may present an unreasonable risk of illness or injury. Highrisk devices, which include implantable pacemakers and deep brain stimulators, are subject to the highest level of premarket scrutiny by the FDA under the premarket approval (PMA) pathway. ${ }^{8}$ The PMA process typically requires that manufacturers conduct at least 1 clinical trial, ${ }^{9}$ with the key trial designed to demonstrate the effectiveness and safety of a device designated by the FDA as the "pivotal" trial in the approval process. High-risk devices may also be approved under the Humanitarian Device Exemption (HDE) program, which applies to devices for rare diseases (affecting $<4000$ patients annually) and mandates studies that demonstrate device safety and probable benefit before approval.

A few federal regulatory initiatives have encouraged greater attention to the application of medical device technology to the needs of pediatric patients. For example, the FDA waives regulatory review fees under the PMA pathway for devices indicated solely for children. ${ }^{10}$ Furthermore, manufacturers have traditionally been prohibited from selling HDE devices for profit. However, in September 2007, Congress passed the Pediatric Medical Device Safety and Improvement Act (PMDSIA), eliminating these profit restrictions on pediatric HDE devices to further stimulate development of medical devices for children. ${ }^{11}$ The statute also established grants for nonprofit pediatric device development consortia. Starting in 2009, PMDSIA required that the FDA track and report to Congress the number and types of devices approved in the preceding year for pediatric use.

In the wake of the enactment of PMDSIA, we identified and analyzed all devices approved for use in pediatric populations to define the current state of trial evidence underpinning pediatric device approvals since 2007. We focused on highrisk device approvals through the PMA and HDE pathways, because these devices present the greatest danger while also offering the potential for substantial therapeutic benefit to children.

\section{METHODS}

\section{Pediatric Device Approvals}

The study cohort included all medical devices indicated for therapeutic use in pediatric patients (including any subpopulation based on age) and approved by the FDA through the PMA or HDE pathways between January 1,2008 , and December 31, 2011. Devices were selected for inclusion on the basis of lists compiled by the FDA for its annual reports to Congress on pediatric medical devices submitted for fiscal years 2008-2011. ${ }^{12}$
These reports provide a complete list of all devices that were approved for use in pediatric patients during the preceding year and were initiated after passage of PMDSIA in 2007. To identify any devices that could have been missed using this approach, we cross-referenced our results with the FDA's electronic databases of all PMA ${ }^{13}$ and HDE ${ }^{14}$ approvals and selected devices for which the label indicated approval for use in patients aged $\leq 21$ years. This age cutoff is based on section 515A of the Federal Food, Drug, and Cosmetic Act, as amended by the FDA Amendments Act of 2007, which defines pediatric patients as those aged $\leq 21$ years at the time of their diagnosis or treatment. ${ }^{15}$ We also identified the subgroup of devices approved specifically for patients aged $<18$ years, because this is a commonly used threshold to define pediatric patients among practitioners. If the label did not specify the minimum approval age but stated that the device was approved for pediatric patients $(N=$ 1 ), we assumed that this statement referred to patients $<18$ years.

\section{Selection of Pivotal Device Trials}

For each device in the cohort, we examined the review summaries compiled by the FDA on the key clinical data that it received from the sponsor and that were used to support the device's approval. From the FDA review summaries, we identified the pivotal clinical trials that supported the intended indication(s) and patient population(s) for which the device was approved. We considered a trial to be pivotal if it was specifically referenced by the FDA review as a pivotal (or "primary" or "major") trial. In 2 (8\%) cases, the pivotal trial was not specifically labeled, and in these cases we considered the pivotal trial to be the one that was the most advanced study to be completed (eg, if results from phase 1 and phase 2 trials were presented, the phase 2 trial was considered pivotal). All devices in our cohort were associated 
with 1 pivotal trial each. Of note, some studies designated as pivotal by the FDA for PMA and HDE applications included observational studies and studies based on disease registries.

\section{Characteristics of Device Trials}

Characteristics of the pivotal trials were abstracted by 1 author (T.J.H.) from the FDA approval order, product label, and review summaries (all data downloaded July 18, 2013). If there were any ambiguities, the data were reviewed by a second author (F.T.B.) and reconciled. We collected information on trial participants including the total number of participants enrolled and their ages. Trial characteristics assessed were based on trial design and methods and included the following: whether participants were randomly assigned, the masking scheme (double-blind, singleblind, post hoc review blind, or openlabel), and the types of comparators used (active comparator, standard of care, placebo with or without crossover, historical control, single-arm/no control). We also recorded the primary end points, as classified by the FDA and the manufacturer, used to assess effectiveness in the pivotal trials (Supplemental Table 2). These were categorized as measures of survival, procedural (or technical) end points (eg, successful implantation rates), or surrogate end points of disease response or progression (eg, reduction in number of postoperative interventions).

Finally, we identified any postmarketing studies required by the FDA as part of the approval order along with the required participant ages. We ran a search for these trials on the FDA's online database of postapproval trials to determine the most recent status of these studies. ${ }^{16}$

\section{Statistical Analysis}

For each device in our sample, we determined the total number of pediatric participants studied in the pivotal trials.
We calculated the median number of pediatric participants per trial across all pediatric devices and for the 2 types of devices. For trials enrolling both adult and pediatric patients, we calculated the proportion of participants reported to be $<18$ years old. For trials that did not report data on the age distribution of participants $(N=6)$, we used the mean, $\mathrm{SD}$, and age range to estimate the maximum number of participants who could have been $<18$ years old assuming a normal distribution in age (eg, $95 \%$ of participants were within 2 SDs of the mean age). Statistical analyses were performed by using Stata (version 12.0; Stata Corp, College Station, TX).

\section{RESULTS}

We identified 25 pediatric devices, 22 (88\%) approved through the PMA process and $3(12 \%)$ via the HDE program. One device, an injectable gel indicated for the treatment of fecal incontinence in patients $\geq 18$ years old, was identified from our manual search of PMA approvals. Three (12\%) of the pediatric devices were approved for use in patients aged $<18$ years ( 1 via the PMA and 2 via the HDE pathway), whereas the remaining 22 (88\%) were approved for use in patients $\geq 18$ years. The most common therapeutic area was cardiovascular (15 devices; $60 \%$ ), followed by obstetrics and gynecology (2 devices; 8\%) and gastroenterology and urology (2 devices; 8\%).

In total, among the 25 trials supporting the approval of devices for use in children, 5058 participants were enrolled and studied. Pivotal trials enrolled a median of 166 participants per trial, with PMA devices enrolling a median of 182 participants (interquartile range [IQR]: 146-265) and HDE devices enrolling a median of 50 patients (IQR: 33-99) per trial. There were 226 (4\%) patients $\leq 21$ years and $196(4 \%)$ patients $<18$ years old enrolled in the pivotal trials. Overall, 11 (44\%) pediatric devices were not studied in any patients $\leq 21$ years, and 21 (84\%) devices were not studied in any patients $<18$ years old.

PMA Devices Indicated for Patients Aged $<18$ years

Among the 22 medical devices approved through the PMA process for use in a pediatric subpopulation, only 1 device was indicated for use in patients $<18$ years. The device (Repel-CV; Pathfinder Cell Therapy, Cambridge), a bioresorbable barrier for postoperative cardiac adhesions, was approved for newborns younger than 1 year. The pivotal trial, which was randomized and single-blinded, enrolled 144 patients with an age range of 2 days to 3 months. The primary end point was a surrogate end point, consisting of the percentage of studydefined interventional surgical sites with severe adhesions at the second sternotomy procedure.

\section{PMA Devices Indicated for Patients Aged $\geq 18$ Years}

For the 21 PMA devices approved in patients $\geq 18$ years, a total of 4732 participants were studied in the pivotal trials. Information on the age range of trial participants was available for 15 devices. The pivotal studies for 11 (11 of $15 ; 73 \%$ ) devices did not enroll any patients $\leq 21$ years, and the median age of the youngest participant was 36 years (IQR: 29-46 years). Three (19\%) trials reported enrolling patients aged $\geq 18$ years, and 1 trial included a minimum age of 16 years. These 4 trials enrolled at most 14 patients who were $\leq 21$ years and at most 3 patients who were $<18$ years.

For the 6 devices without information on the age ranges of trial participants, the median age for patients enrolled in the pivotal studies was 43 years (IQR: 34-60 years). The exclusion criteria in all of these studies ruled out any participants $<18$ years.

Table 1 shows the trial characteristics for the 21 pediatric PMA devices indicated in patients aged $\geq 18$ years. A 
total of 8 (8 of 21; 38\%) pivotal trials for the pediatric-approved devices were randomized. In 7 of these trials, the device was compared with standard of care or placebo (eg, sham control), whereas 1 trial used an intrapatient control design with crossover from treatment to placebo. Treatment group assignment was masked in 6 (6 of 21; 29\%) trials, with double-blinding in 5 trials and single-blinding in 1 trial. Among the 13 nonrandomized studies, 4 compared the treatment group with a historical comparator and 9 were single-arm studies. The majority (18 of $21 ; 86 \%)$ of pivotal trials used surrogate primary end points, whereas 3 measured survival (30-day rupture-free survival, proportion of patients surviving either to cardiac transplantation or device support, and all-cause mortality).

\section{HDE Devices}

Three pediatric devices intended to treat rare diseases were approved through the HDE pathway, including an arteriotomy system, a pulmonary valve prosthesis, and a diaphragmatic pacing system. One of the HDE devices was approved for children aged $\geq 13$ years, and the label for another stated that the device was approved for both pediatric and adult patients but did not provide specific pediatric ages. The third HDE device was approved in patients $\geq 18$ years.

The 3 pivotal trials associated with the pediatric HDE devices enrolled a total of 182 participants: 68 (37\%) were $\leq 21$ years and an estimated 49 (27\%) were $<18$ years old. ${ }^{17}$

\section{Postmarketing Studies}

Nineteen (76\%) pediatric devices were approved with postmarketing study requirements (17 PMA and 2 HDE devices). For 3 of these, the FDA required that the sponsor include pediatric participants within the age range for which the device was approved. All 3 of these devices were approved for use in patients $<18$ years, including 1 device approved for use in neonates.

As of September 2013, the FDA reported that, of the 19 required postmarketing studies, 3 (18\%) were complete with the final study results submitted, 6 (35\%) had adequate progress toward prespecified study milestones, and 2 (12\%) were terminated because of withdrawn devices. Six (35\%) postmarketing studies were reported to have made inadequate progress, of which 4 had

TABLE 1 Characteristics of Pivotal Trials for Pediatric PMA Devices Approved for Patients Aged $\geq 18$ Years

\begin{tabular}{lc}
\hline \multicolumn{1}{c}{ Characteristics } & Pivotal PMA Trials $(N=21)^{\text {a }}$ \\
\hline Participants, median (IQR), $n$ & $182(150-276)$ \\
Age, median (IQR), y & $36(29-49)$ \\
Randomly assigned, $n(\%)$ & $8(38)$ \\
Comparator, $n$ (\%) & \\
Active or placebo & $3(14)$ \\
Placebo & $5(24)$ \\
Historical control & $4(19)$ \\
None & $9(43)$ \\
Blinding, $n$ (\%) & $6(29)$ \\
Double-blind & $5(24)$ \\
Single-blind & $1(5)$ \\
Open-label & $15(71)$ \\
Primary trial effectiveness end point, $n(\%)$ & \\
Survival & $3(14)$ \\
Procedural success ${ }^{b}$ & $4(19)$ \\
Surrogate end points & $14(67)$ \\
\hline
\end{tabular}

Due to rounding, proportions may not sum to 100 .

a These 21 pivotal trials were related to 21 PMA devices.

b Procedural success was defined as the proportion of implantation success.

missed enrollment deadlines and 2 had follow-up rates of $<80 \%$. All 3 postmarketing studies that required pediatric patient enrollment were reported to have made inadequate progress. The status of 2 postmarketing studies (both for HDE devices) was not reported in the FDA's electronic database.

\section{DISCUSSION}

In this cohort study of FDA device approvals, almost all devices approved for pediatric patients were studied only in patients $\geq 18$ years old and were approved on the basis of nonrandomized, open-label trials with surrogate effectiveness end points.

Our results suggest that most of the recent pediatric device development activity has been by manufacturers who have developed devices primarily for adults. They have obtained pediatric approval on the basis of study in a small age range of patients not typically considered pediatric (18-21 years), and the majority of sponsors assessed their products exclusively in adults. The testing and approval of high-risk medical devices in pediatric populations $<18$ years old remains extremely uncommon. Because PMDSIA also established grants for nonprofit pediatric device development consortia, it is possible that, in the future, these consortia will contribute to increased numbers of pediatric device approvals. ${ }^{18}$ In addition, in February 2013, the FDA issued draft guidance ${ }^{19}$ and a proposed rule ${ }^{20}$ to standardize the submission of pediatric use data from manufacturers. The FDA stated that it intends to use these data to "determine unmet pediatric needs in medical device development ... [and] promote new device development and proper labeling of existing medical devices for pediatric use." If promulgated, this guidance may also lead to improved data to guide clinical decision-making around pediatric devices. 
Our findings have practical implications for physicians and their patients. Both deserve rigorous evidence to make informed treatment decisions that adequately weigh the benefits and risks of a device. Randomized controlled trials are considered the gold standard for clinical investigations, because they minimize potential sources of bias when assessing a cause-effect hypothesis. ${ }^{21}$ Although such trials are the standard for most drug approvals, they are less frequently required as a precondition for approval of medical devices among patients in all age groups, ${ }^{22}$ which is concerning because many devices are implanted and are more difficult to discontinue than drugs. ${ }^{23}$ Even when there is good evidence for the safety and effectiveness of a device in adults, the clinical outcomes and safety profiles may differ between adult and pediatric populations.

There are a number of potential reasons for the low numbers of pediatric patients studied in these trials and for the trial characteristics we observed. Pediatric device trials have a smaller potential pool of trial participants as well as fewer suitable control patients. ${ }^{24}$ Devices approved through the HDE pathway, unlike those approved via the PMA program, are statutorily exempt from the effectiveness requirements of PMA devices and must instead demonstrate "probable benefit"; in addition, the difficulties in recruiting potential trial participants are magnified in cases of rare diseases..$^{25}$ Our findings are consistent with the relative paucity of rigorous clinical evidence for pediatric use of many approved drugs. ${ }^{26}$ In this case, the limited study of pediatric patients has been attributed to a more complicated consent process, lower pediatric disease burden, and poor incentives for industry in terms of returns on investment for pediatric drugs. ${ }^{27,28}$ Additionally, most devices are developed by small companies that may have fewer resources or experience to conduct rigorous clinical studies, ${ }^{29}$ although most devices (13 of 23; $57 \%$ ) in our cohort were developed by companies with gross revenues of US $\$ 1$ billion or more annually.

To supplement premarket trial data, it is generally agreed that continued active study of most high-risk devices is necessary after approval. The FDA has recently committed to develop a comprehensive device surveillance system. ${ }^{30}$ Consistent with that mission, we found that most of the pediatric devices were approved with postmarketing study requirements. However, only 3 approvals were contingent on the sponsor studying the device in the approved pediatric subpopulation (all thus far delayed). Unlike pediatric drug trials, there are currently no special incentives for manufacturers to conduct postapproval studies of medical devices in children. ${ }^{31}$ In 2007, the FDA was authorized to require postmarketing studies longer than 36 months for pediatric devices. It is possible, therefore, that more of these devices will be approved in the future with mandated long-term postmarketing studies in children.
A limitation of our study is that data were primarily obtained from publicly available documents that may have omitted clinical data and information on postmarketing study commitments. However, the PMA and HDE review dockets are required by rule to include the "summaries, conclusions, and results of all clinical experience or investigations (whether adverse or supportive) ... that are relevant to an assessment of the risks and probable benefits of the device."32 Although we focused on pediatric device approvals after enactment of PMDSIA, it is possible that some of the trials for the devices in our sample were designed before passage of the act and further improvements may still occur in terms of pediatric representation, which were not captured in our cohort. However, the approvals of all of the devices studied reflect current FDA requirements.

\section{CONCLUSIONS}

The vast majority of recently introduced pediatric devices were approved on the basis of study in patients $\geq 18$ years old. Most pediatric medical devices were studied in nonrandomized, openlabel studies, and few postmarketing studies required further surveillance of device safety and effectiveness in children. Future efforts to address the lack of evidence to guide the use of devices in children should place greater emphasis on studying the devices in pediatric patients both in premarket and postmarketing trials.

\section{REFERENCES}

1. US Food and Drug Administration. FY 2014 Congressional budget request: justification of estimates for appropriations committees. Available at: www.fda.gov/downloads/ AboutFDA/ReportsManualsForms/Reports/ BudgetReports/UCM349751.pdf. Accessed August 6, 2013
2. Beekman RH III, Duncan BW, Hagler DJ, et al; Workgroup on Pediatric Cardiac Devices, Section on Cardiology and Cardiac Surgery, American Academy of Pediatrics. Pathways to approval of pediatric cardiac devices in the United States: challenges and solutions. Pediatrics. 2009;124(1). Available at: www.pediatrics.org/cgi/content/full/124/ $1 / \mathrm{e} 155$

3. Cecchin F, Atallah J, Walsh EP, Triedman JK, Alexander ME, Berul $\mathrm{Cl}$. Lead extraction in pediatric and congenital heart disease patients. Circ Arrhythm Electrophysiol. 2010;3(5):437-444 
4. Atallah J, Erickson CC, Cecchin F, et al; Pediatric and Congenital Electrophysiology Society (PACES). Multi-institutional study of implantable defibrillator lead performance in children and young adults: results of the Pediatric Lead Extractability and Survival Evaluation (PLEASE) study. Circulation. 2013;127 (24):2393-2402

5. McElhinney DB, Bergersen L, Marshall AC. In situ fracture of stents implanted for relief of pulmonary arterial stenosis in patients with congenitally malformed hearts. Cardiol Young. 2008;18(4):405-414

6. Breinholt JP, Nugent AW, Law MA, Justino $\mathrm{H}$, Mullins CE, Ing FF. Stent fractures in congenital heart disease. Catheter Cardiovasc Interv. 2008;72(7):977-982

7. Sutherell JS, Hirsch R, Beekman RH III. Pediatric interventional cardiology in the United States is dependent on the off-label use of medical devices. Congenit Heart Dis. 2010;5(1):2-7

8. Kramer DB, Xu S, Kesselheim AS. Regulation of medical devices in the United States and European Union. N Engl J Med. 2012;366(9): 848-855

9. Kaplan AV, Baim DS, Smith JJ, et al. Medical device development: from prototype to regulatory approval. Circulation. 2004;109 (25):3068-3072

10. Medical Device User Fee and Modernization Act of 2002, Pub L No. 107-250

11. Pediatric Medical Device Safety and Improvement Act of 2007, Pub L No. 110-85

12. US Food and Drug Administration. Pediatric report to Congress, FY 2008-2011. Available at: www.fda.gov/MedicalDevices/ProductsandMedicalProcedures/ucm 135104.htm. Accessed July 18, 2013

13. US Food and Drug Administration. Premarket approval. Available at: www.accessdata.fda. gov/scripts/cdrh/cfdocs/cfpma/pma.cfm. Accessed July 18, 2013

14. US Food and Drug Administration. Listing of CDRH humanitarian device exemptions. Available at: www.fda.gov/medicaldevices/ productsandmedicalprocedures/deviceapprovalsandclearances/hdeapprovals/ucm 161827.htm. Accessed July 18, 2013

15. Federal Food, Drug, and Cosmetic Act, $\S 515$ (a)

16. US Food and Drug Administration. Postapproval studies. Available at: www.accessdata. fda.gov/scripts/cdrh/cfdocs/cfpma/pma_pas. cfm. Accessed July 18, 2013

17. Sapirstein JS. FDA Pediatric Advisory Committee. Medtronic Melody Transcatheter Pulmonary Valve (TPV) presentation. Available at: www.fda.gov/downloads/AdvisoryCommittees/CommitteesMeetingMaterials/ PediatricAdvisoryCommittee/UCM319365.pdf

18. Ulrich LC, Joseph FD, Lewis DY, Koenig RL. FDA's pediatric device consortia: national program fosters pediatric medical device development. Pediatrics. 2013;131(5):981-985

19. US Food and Drug Administration. Draft guidance: providing information about pediatric uses of medical devices under section 515A of the Federal Food, Drug, and Cosmetic Act. Available at: www.fda.gov/downloads/ MedicalDevices/DeviceRegulationandGuidance/ GuidanceDocuments/UCM339465.pdf. Accessed July 2, 2013

20. US Food and Drug Administration. "Proposed rule: pediatric uses of devices; requirement for submission of information on pediatric subpopulations that suffer from a disease or condition that a device is intended to treat, diagnose, or cure (finalized in January 10, 2014)". Fed Regist. 2013; 78(33):11612-11617

21. Sibbald B, Roland M. Understanding controlled trials: why are randomised controlled trials important? BMJ. 1998;316(7126): 201

22. Garber AM. Modernizing device regulation. N Engl J Med. 2010;362(13):1161-1163

23. Dhruva SS, Bero LA, Redberg RF. Strength of study evidence examined by the FDA in premarket approval of cardiovascular devices. JAMA. 2009;302(24):2679-2685

24. Dolcimascolo F. Pediatric cardiac devicesan FDA pediatrician's perspective of the challenges and potential solutions. $J$ Cardiovasc Trans/ Res. 2009;2(2):147-149

25. Hwang TJ, Carpenter D, Kesselheim AS. Assessment of US pathway for approving medical devices for rare conditions. BMJ. 2014;348:g217

26. Martinez-Castaldi C, Silverstein M, Bauchner $H$. Child versus adult research: the gap in high-quality study design. Pediatrics. 2008; 122(1):52-57

27. Caldwell PH, Murphy SB, Butow PN, Craig JC. Clinical trials in children. Lancet. 2004; 364(9436):803-811

28. Steinbrook R. Testing medications in children. N Engl J Med. 2002;347(18):1462-1470

29. Campbell B, Stainthorpe AC, Longson CM. How can we get high quality routine data to monitor the safety of devices and procedures? BMJ. 2013;346:f2782

30. US Food and Drug Administration. Strengthening our national system for postmarket device surveillance. 2012. Available at: www. fda.gov/AboutFDA/CentersOffices/OfficeofMedicalProductsandTobacco/CDRH/CDRHReports/ ucm301912.htm. Accessed July 2, 2013

31. Li JS, Eisenstein EL, Grabowski HG, et al. Economic return of clinical trials performed under the pediatric exclusivity program. JAMA. 2007;297(5):480-488

32. Federal Register (codified as 21 CFR $\S \S 814.104,814.122)$ 